\title{
Reduced Paraoxonase 1 Activity as a Marker for Severe Coronary Artery Disease
}

\author{
Chiyan Zhou, ${ }^{1}$ Jia Cao, ${ }^{1}$ Liang Shang, ${ }^{1}$ Chuanfeng Tong, ${ }^{2}$ Hanling Hu, ${ }^{3}$ Hui Wang, \\ Daping Fan, ${ }^{5}$ and Hong $\mathrm{Yu}^{1,4}$ \\ ${ }^{1}$ Department of Biochemistry and Molecular Biology, Wuhan University School of Medicine, Wuhan, Hubei 430071, China \\ ${ }^{2}$ Cardiology Division, Wuhan University Zhongnan Hospital, Wuhan 430071, China \\ ${ }^{3}$ Clinical Biochemistry Laboratory, Wuhan University Zhongnan Hospital, Wuhan 430071, China \\ ${ }^{4}$ Research Center of Food and Drug Evaluation, Wuhan University School of Medicine, Wuhan 430071, China \\ ${ }^{5}$ Department of Cell Biology and Anatomy, School of Medicine, University of South Carolina, Columbia, SC 29208, USA
}

Correspondence should be addressed to Hong Yu; yu.hong@whu.edu.cn

Received 23 February 2013; Revised 13 June 2013; Accepted 17 June 2013

Academic Editor: Benoit Dugue

Copyright (C) 2013 Chiyan Zhou et al. This is an open access article distributed under the Creative Commons Attribution License, which permits unrestricted use, distribution, and reproduction in any medium, provided the original work is properly cited.

\begin{abstract}
Paraoxonase-1 (PON1), a high-density-lipoprotein- (HDL-) associated enzyme, has the potential to protect against atherogenesis. We examine the relationships between plasma PON1 activity and the progression of atherosclerosis as well as coronary artery disease (CAD). Fasting blood samples were collected from female apolipoprotein E-deficient (apoE ${ }^{-/-}$) mice and 149 patients undergoing coronary angiography for the biochemical parameters measurement. The severity of CAD was defined using angiographic Gensini score (GSS). Compared to 3-month-old apoE ${ }^{-/-}$mice, aged mice had significantly lower PON1 activity, which is negatively correlated with the size of atherosclerotic lesion and plasma interleukin-6 (IL-6) and tumor necrosis factor $\alpha$ (TNF- $\alpha$ ) levels. In study patients, $\mathrm{PON} 1$ activity was correlated with age, sex, and HDL-cholesterol, apolipoprotein AI, and high-sensitivity C-reactive protein (hs-CRP) levels and was significantly lower in CAD group than that in non-CAD control group. Interestingly, PON1 activity in severe CAD group (GSS $>40$ ) was further significantly reduced compared to those in mild and moderate subgroups (GSS $\leq 40)$ $(P<0.01)$. There is a significant correlation between PON1 activity and the severity of CAD as assessed by GSS $(r=-0.393$, $P<0.001)$. PON1 activity may be a potential biomarker for the severity of CAD.
\end{abstract}

\section{Introduction}

Atherosclerosis is characterized by accumulation of lipids and inflammatory cells in the artery wall and is the cause of coronary artery disease (CAD). Although the mechanisms are still not entirely resolved, previous studies have indicated that dyslipidemia, enhanced oxidative stress, and inflammation result in the development of atherosclerosis and its complications, CAD $[1,2]$.

There is increasing evidence that oxidized low-density lipoprotein (LDL) has a key role in the initiation and progression of atherosclerosis; high-density lipoprotein (HDL) has an antiatherogenic role in part by its antioxidative and anti-inflammatory properties through preventing LDL oxidation [3]. The HDL-associated enzyme paraoxonase-1 (PON1), a calcium-dependent esterase, is largely responsible for the antioxidant and anti-inflammatory actions of HDL due to its ability to hydrolyze oxidized phospholipids [4, 5]. Many experiments have shown that knockout of PON1 gene is proinflammatory and pro-atherogenic by increasing oxidized LDL and cell adhesive molecules, while transgene or overexpression of $\mathrm{PON} 1$ is antiatherogenic by decreasing LDL oxidation and inflammatory status [6-9]. Recent metaanalyses of clinical studies suggested that lower plasma PON1 activity is associated with increased CAD risk $[10,11]$ and that decreased PON1 activity might be a factor responsible for the acceleration of CAD in type 2 diabetes mellitus [12]. It has been shown that PON1 activity is affected by both genetic 
polymorphisms and environmental factors including age, gender, lifestyle, and pharmaceutical interventions [13, 14]. Despite the current knowledge, the function and mechanism of PON1 in the initiation and progression of atherosclerosis warrant further investigation.

In this study, we first used apolipoprotein E-deficient $\left(\right.$ apoE $^{-/-}$) mice to investigate the change of plasma PON1 activity during the progression of atherosclerosis. $\mathrm{ApoE}^{-/-}$ mice have been widely used in atherosclerosis studies because they develop severe hypercholesterolemia and spontaneous atherosclerotic lesions with age. Next, we extended our study by examining the relationship between plasma PON1 activity and the severity of coronary artery stenosis in Chinese CAD patients confirmed by coronary angiography.

\section{Materials and Methods}

2.1. Experimental Subjects. ApoE ${ }^{-/-}$mice (on C57BL/6 background) were obtained from Jackson Laboratories and housed in microisolator cages on a rodent chow diet. Animal care and experimental procedures were performed under the regulations of the Animal Care Center of Wuhan University, in accordance with the guidelines laid down by the National Institutes of Health of the United States. Only female mice with different age were used for this study.

The clinical study was performed in the Cardiology Division of Wuhan University Zhongnan Hospital in Hubei province, China. Patients (105 males and 44 females; mean age $61.1 \pm 9.0$ yrs) were recruited for this study when they visited the hospital for coronary angiography from September 2011 to May 2012. The percent stenosis of the coronary artery was determined by the hand-held caliper measurement. Patients with angiographic CAD $(n=118)$ were defined as the presence of stenosis $\geq 50 \%$ of the luminal diameter in at least one coronary artery. The severity of CAD was assessed using angiographic Gensini score (GSS) [15]. The GSS was calculated for each coronary stenosis based on the degree of luminal narrowing and its localization. Mild atherosclerosis was classified as a GSS $\leq 10$, moderate atherosclerosis as a GSS $>10$ and $\leq 40$, and severe atherosclerosis as a GSS $>40$ [16]. Those taking lipid-lowering drugs and antioxidants or having suspected infectious conditions, autoimmune-related disease, peripheral artery disease, or renal and hepatic diseases were excluded. Written informed consent was obtained from all participants before collecting blood samples and general information regarding health, medical history, and lifestyle habits. The study was approved by the Medical Ethics Committee of Wuhan University Zhongnan Hospital, confirmed with the Declaration of Helsinki of the World Medical Association.

Blood samples with or without heparin were collected after fasting overnight. Mouse blood was collected by retroorbital venous plexus puncture under the anaesthetization with $3 \%$ isoflurane. Human blood samples were obtained before coronary angiography. The plasma or serum was immediately separated by centrifugation of $4000 \times \mathrm{g} 10 \mathrm{~min}$ at $4^{\circ} \mathrm{C}$, and then serum was immediately aliquoted and stored at $-80^{\circ} \mathrm{C}$.
2.2. Biochemical Analyses. PON1 activity was measured spectrophotometrically using paraoxon (O,O-diethyl-O-(4nitrophenyl) phosphate, Sigma Chemical Co., MO, USA) as substrate. Five microliters of serum or plasma without heparin mixed with $95 \mu \mathrm{L}$ assay buffer $(2.0 \mathrm{~mol} / \mathrm{L} \mathrm{NaCl}$, $100 \mathrm{mmol} / \mathrm{L}$ Tris- $\mathrm{HCl}, 2.0 \mathrm{mmol} / \mathrm{L} \mathrm{CaCl}_{2}, \mathrm{pH}$ 8.5) was placed in a 96-well plate, and then $100 \mu \mathrm{L}$ paraoxon solution $(2.4 \mathrm{mmol} / \mathrm{L}$ in assay buffer) was added into each well. The rate of generation of product p-nitrophenol was monitored by measuring the increase of absorbance at $405 \mathrm{~nm}$ at $25^{\circ} \mathrm{C}$ within $4 \mathrm{~min}$. PON1 activity was calculated from the molar extinction coefficient $\left(17,000 \mathrm{M}^{-1} \cdot \mathrm{cm}^{-1}\right)$. One unit of PON1 activity was defined as $1 \mathrm{nmol}$ of $\mathrm{p}$-nitrophenol formed per minute under the previous conditions and expressed as $\mathrm{U} / \mathrm{mL}$ serum.

Plasma total cholesterol (TC) and triglycerides levels were measured by enzymatic colorimetric assays (Prodia diagnostics, Boetzingen, Germany). HDL-cholesterol (HDLC) was determined after precipitation of the apolipoprotein B-containing lipoproteins, and LDL-cholesterol (LDL-C) was calculated using Friedewald formula (Sekisui, Tokyo, JP). Lipoprotein (a), apolipoprotein AI (apoAI), and apolipoprotein B100 (apoB100) were detected by immunoturbidimetric assays (Prodia diagnostics, Boetzingen, Germany). All the assays were performed blindly. Serum tumor necrosis factor alpha (TNF- $\alpha$ ) and interleukin-6 (IL-6) levels of mice were determined using an enzyme-linked immunosorbent assay (ELISA) kit according to the manufacturer's instructions (eBioscience, San Diego, USA). Each sample was assayed in triplicate, and the intra-assay coefficient of variation was less than $10 \%$. The concentration of high-sensitivity C-reactive protein (hs-CRP) in human plasma was determined on an Olympus analyzer by a high sensitivity latex-enhanced immunoturbidimetry assay using Nanopia CRP kit (Sekisui, Tokyo, JP) with standards and controls supplied by the manufacturer.

2.3. Quantification of Atherosclerotic Lesions in Mice. The proximal aortas were collected from 3-, 6-, 12-, or 18-month old $\mathrm{apoE}^{-/-}$mice fed a regular chow diet after the mice were sacrificed by an overdose of isoflurane. The extent of atherosclerosis was examined both in oil-red O-stained crosssections of the proximal aorta (15 alternate $10 \mu \mathrm{m}$ cryosections) and by en face analysis using the KS300 imaging system as described previously [17].

2.4. Statistical Analysis. Statistical analysis was performed using the SPSS 17.0 (SPSS Inc., Chicago, IL, USA). Continuous variables were expressed as mean \pm SD (standard deviation) or median with 25 th and 75 th percentiles. The Student's unpaired $t$-test for parametric variables or the Mann-Whitney $U$ test for nonparametric variables was used to assess difference between two groups. Difference among multiple groups was analyzed by one-way analysis of variance (ANOVA) followed by pairwise comparison with the method of Fisher's LSD. Univariate correlations of PON1 activity with risk factors were assessed by Pearson or Spearman 

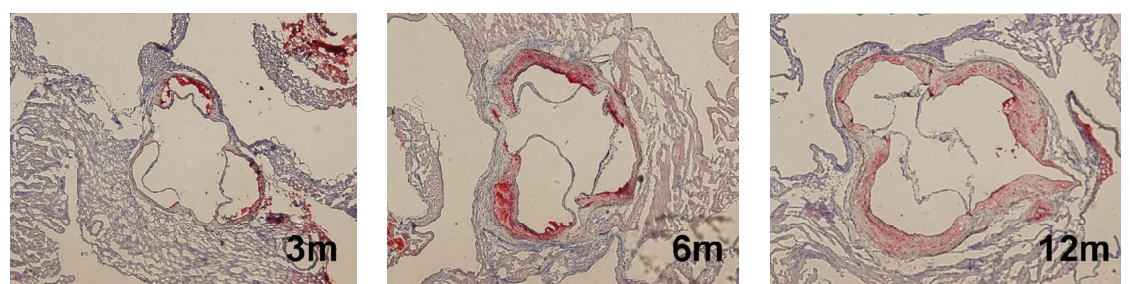

(a)
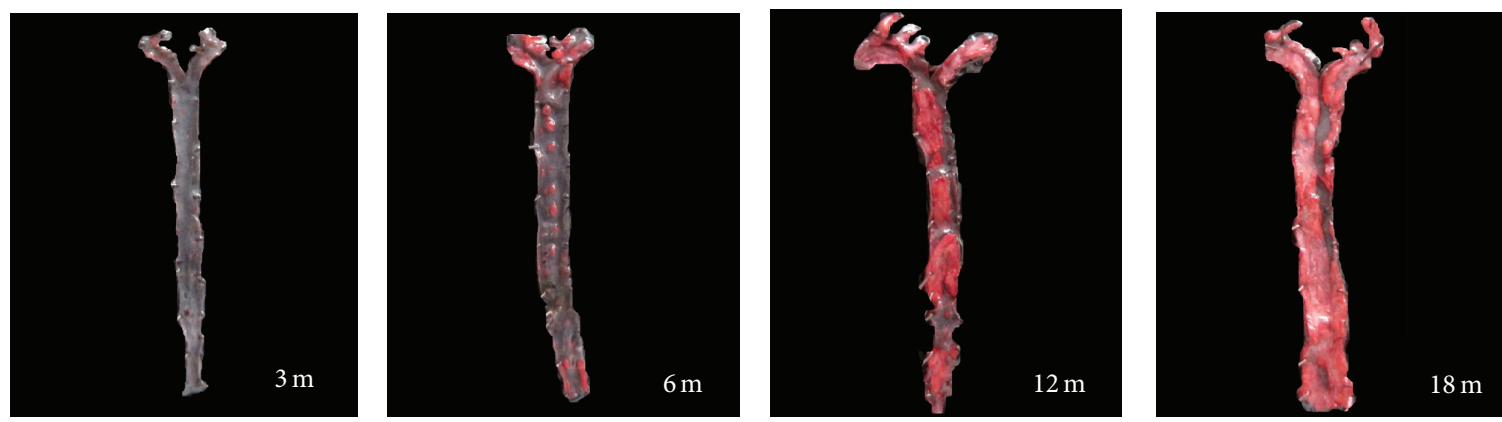

(b)

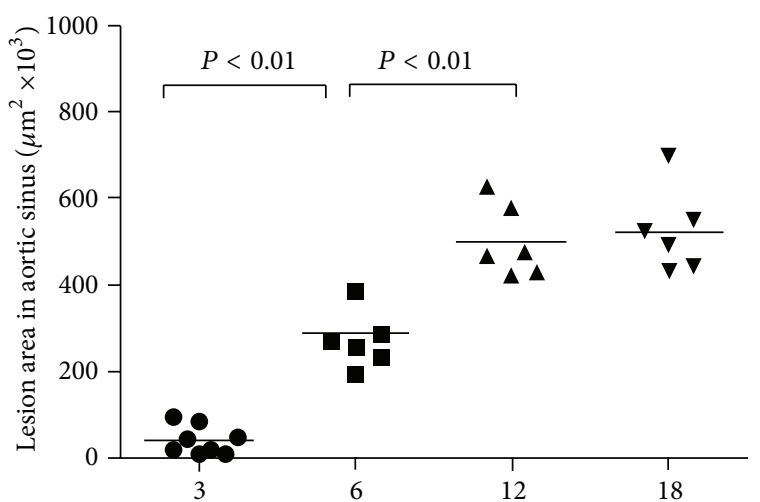

$(\mathrm{m})$

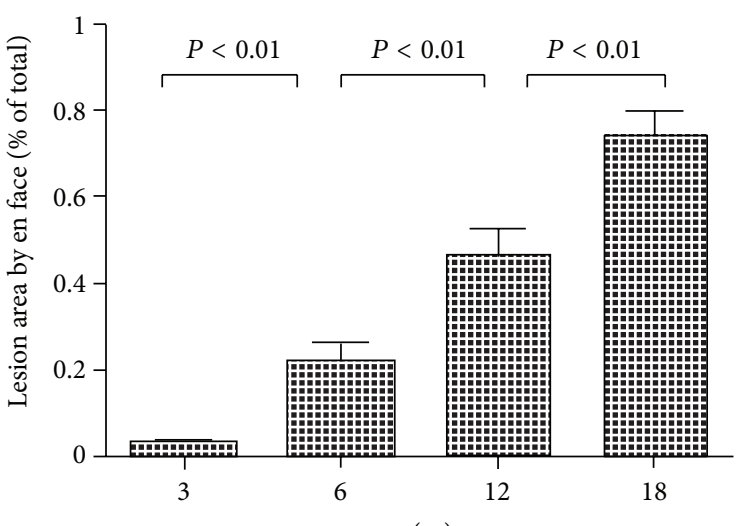

$(\mathrm{m})$

(c)

(d)

FIGURE 1: Atherosclerotic lesion areas in proximal aortas of $\mathrm{apoE}^{-/-}$mice with different ages. (a) Representative atherosclerotic lesions in cross-sections of proximal aortas stained with oil-red O. (b) Representative en face aortas from 3 months to 18 months. Quantitation of the mean lesion area of aortic sinus (c) and percentage lesion area in the aorta by en face analysis (d).

correlation. All tests were two tailed, and $P$ values of $<0.05$ were considered statistically significant.

\section{Results}

$\mathrm{ApoE}^{-/-}$mouse is a widely used animal model for atherosclerosis study. Aged apoE $\mathrm{E}^{-/-}$mice develop advanced atherosclerotic lesions similar to those in human arteries. In this study, progressive atherosclerotic lesions were observed in apoE ${ }^{-/-}$mice with increased age on a regular chow diet (Figure 1). The first sign of lesions was present in the aortic root and aortic arch at 3 months of age, and then the lesions of various stages widely appeared and were gradually accelerated throughout the arterial tree of elder mice. With the progressive atherosclerosis, hypercholesterolemia was also significantly aggravated in elder mice, whereas triglycerides levels were not significantly changed (Table 1). It is notable that serum PON1 activity in apoE ${ }^{-/-}$mouse gradually decreased while atherosclerosis progressed. The lower activity of PON1 correlated with more severe lesions, and decrease in PON1 activity was accompanied with the increase of other known inflammatory factors, such as TNF- $\alpha$ and IL-6.

We next examined the correlation of PON1 activity with the development of CAD in Chinese Han population. A total of 118 CAD patients (aged $61.6 \pm 9.2 \mathrm{yrs}, 29.7 \%$ women) and 31 non-CAD patients (aged $59.3 \pm 8.3$ yrs, $29.0 \%$ women) documented by the angiographic reports were included in the analysis. According to Gensini score (GSS), among the 118 CAD patients, 27 of them (22.9\%) were mild (GSS $\leq$ $10), 43(36.4 \%)$ were moderate (GSS $>10$ and $\leq 40$ ), and $48(40.7 \%)$ were severe CAD patients. The clinical and biochemical parameters of the study patients were shown in 
TABLE 1: Plasma PON1 activity and biochemical index in apo: ${ }^{-/-}$mice with different ages.

\begin{tabular}{lcccc}
\hline Parameters & $\begin{array}{c}3 \text { months } \\
(n=8)\end{array}$ & $\begin{array}{c}6 \text { months } \\
(n=8)\end{array}$ & $\begin{array}{c}12 \text { months } \\
(n=7)\end{array}$ & $\begin{array}{c}18 \text { months } \\
(n=6)\end{array}$ \\
\hline TC $(\mathrm{mmol} / \mathrm{L})$ & $13.34 \pm 1.24$ & $13.73 \pm 1.03$ & $14.74 \pm 0.90^{* \#}$ & $15.64 \pm 0.79^{* * \#}$ \\
Triglycerides $(\mathrm{mmol} / \mathrm{L})$ & $1.91 \pm 0.38$ & $1.95 \pm 0.41$ & $1.63 \pm 0.18$ & $1.72 \pm 0.20$ \\
TNF- $\alpha(\mathrm{pg} / \mathrm{mL})$ & $74.1 \pm 21.4$ & $124.4 \pm 24.8^{* *}$ & $150.9 \pm 23.9^{* *}$ & $177.3 \pm 10.0^{* * \# \# \Delta}$ \\
IL-6 $(\mathrm{pg} / \mathrm{mL})$ & $92.3 \pm 11.8$ & $114.0 \pm 14.7^{*}$ & $111.0 \pm 22.8$ & $154.9 \pm 17.9^{* * \# \# \Delta}$ \\
PON1 activity $(\mathrm{U} / \mathrm{mL})$ & $121.9 \pm 8.1$ & $108.6 \pm 8.2^{*}$ & $96.6 \pm 13.2^{* * \#}$ & $80.6 \pm 6.7^{* * \# \# \Delta}$ \\
\hline
\end{tabular}

Data are given as mean $\pm \mathrm{SD} .{ }^{*} P<0.05,{ }^{* *} P<0.01$, compared with $3 \mathrm{~m}$ group; ${ }^{\#} P<0.05,{ }^{\# \#} P<0.01$, compared with $6 \mathrm{~m}$ group; ${ }^{\triangle} P<0.05,{ }^{\Delta \triangle} P<0.01$, compared with $12 \mathrm{~m}$ group.

TABLE 2: Clinical and laboratory characteristics of the participants in the study.

\begin{tabular}{|c|c|c|c|c|c|c|}
\hline Parameters & $\begin{array}{l}\text { Non-CAD } \\
\text { patients } \\
(n=31)\end{array}$ & $\begin{array}{l}\text { All CAD } \\
\text { patients } \\
(n=118)\end{array}$ & $P$ & $\begin{array}{c}\text { Mild CAD } \\
(\text { Score } \leq 10, n=27)\end{array}$ & $\begin{array}{c}\text { Moderate CAD } \\
(10<\text { score } \leq 40, \\
n=43)\end{array}$ & $\begin{array}{c}\text { Severe CAD } \\
(\text { Score }>40, n=48)\end{array}$ \\
\hline Gensini score & $2.08 \pm 1.72$ & $37.03 \pm 30.89$ & $<0.001^{* *}$ & $6.67 \pm 2.03$ & $20.17 \pm 7.03^{\# \#}$ & $69.20 \pm 21.95^{\# \# \Delta}$ \\
\hline Age (yrs) & $59.3 \pm 8.3$ & $61.6 \pm 9.2$ & 0.205 & $61.4 \pm 10.7$ & $61.7 \pm 9.4$ & $61.6 \pm 8.3$ \\
\hline Sex (male/female) & $22 / 9$ & $83 / 35$ & 0.946 & $15 / 12$ & $31 / 12$ & $37 / 11$ \\
\hline Smoking & $12(38.7 \%)$ & $36(30.5 \%)$ & 0.597 & $7(25.9 \%)$ & $10(23.3 \%)$ & $19(39.6 \%)$ \\
\hline Diabetes $(n(\%))$ & $3(9.7 \%)$ & $42(35.6 \%)$ & $0.005^{* *}$ & $8(29.6 \%)$ & $13(30.2 \%)^{*}$ & $21(43.8 \%)^{* *}$ \\
\hline Hypertention $(n(\%))$ & $12(38.7 \%)$ & $79(66.9 \%)$ & $0.004^{* *}$ & $20(74.1 \%)^{* *}$ & $25(58.1 \%)$ & $34(70.8 \%)^{* *}$ \\
\hline Triglycerides (mmol/L) & $1.14(0.95 \sim 1.61)$ & $1.50(1.14 \sim 2.35)$ & $0.009^{* *}$ & $2.32(1.23 \sim 3.85)^{* *}$ & $1.46(1.20 \sim 2.07)$ & $1.53(1.04 \sim 2.23)$ \\
\hline $\mathrm{TC}(\mathrm{mmol} / \mathrm{L})$ & $4.16 \pm 0.80$ & $4.42 \pm 1.29$ & 0.285 & $4.65 \pm 1.36$ & $4.33 \pm 1.35$ & $4.38 \pm 1.22$ \\
\hline LDL-C (mmol/L) & $2.63 \pm 0.70$ & $2.70 \pm 1.00$ & 0.711 & $2.53 \pm 0.94$ & $2.76 \pm 1.11$ & $2.74 \pm 0.94$ \\
\hline $\mathrm{HDL}-\mathrm{C}(\mathrm{mmol} / \mathrm{L})$ & $1.15 \pm 0.28$ & $0.95 \pm 0.22$ & $<0.001^{* *}$ & $0.99 \pm 0.25^{*}$ & $0.92 \pm 0.23^{* *}$ & $0.94 \pm 0.18^{* *}$ \\
\hline HDL-C/LDL-C & $0.47 \pm 0.18$ & $0.39 \pm 0.13$ & $0.004^{* *}$ & $0.39 \pm 0.11$ & $0.38 \pm 0.13$ & $0.37 \pm 0.13$ \\
\hline ApoAI (g/L) & $1.22 \pm 0.20$ & $1.09 \pm 0.24$ & $0.007^{* *}$ & $1.21 \pm 0.30$ & $1.08 \pm 0.25^{* \#}$ & $1.03 \pm 0.17^{* * \# \#}$ \\
\hline ApoB100 (g/L) & $0.87 \pm 0.22$ & $0.90 \pm 0.27$ & 0.597 & $0.86 \pm 0.22$ & $0.91 \pm 0.33$ & $0.91 \pm 0.25$ \\
\hline ApoAI/ApoB100 & $1.51 \pm 0.41$ & $1.27 \pm 0.38$ & $0.002^{* *}$ & $1.40 \pm 0.37$ & $1.22 \pm 0.34$ & $1.21 \pm 0.37$ \\
\hline $\operatorname{Lipoprotein}(\mathrm{a})(\mathrm{mg} / \mathrm{dL})$ & $84.8(40.9 \sim 176.4)$ & $78.6(39.5 \sim 173.1)$ & 0.912 & $66.7(21.9 \sim 118.7)$ & $87.5(39.7 \sim 167.0)$ & $94.9(41.3 \sim 268.4)$ \\
\hline PON1 activity (U/mL) & $506.4 \pm 118.8$ & $368.2 \pm 120.0$ & $<0.001^{* *}$ & $404.3 \pm 117.6^{* *}$ & $403.8 \pm 113.6^{* *}$ & $316.0 \pm 109.5^{* * \# \# \Delta}$ \\
\hline hs-CRP (mg/L) & $1.60(0.31 \sim 2.56)$ & $2.40(0.99 \sim 6.17)$ & $0.003^{* *}$ & $1.58(0.80 \sim 4.07)$ & $1.53(0.81 \sim 4.46)$ & $4.30(2.00 \sim 9.02)^{* * \# \# \Delta}$ \\
\hline
\end{tabular}

Data for continuous variables as mean \pm SD or median (25th and 75th percentiles); data presented as numbers (percentages) of participants.

${ }^{*} P<0.05,{ }^{* *} P<0.01$, compared with non-CAD group; ${ }^{\#} P<0.05$, ${ }^{\# \#} P<0.01$, compared with mild stenosis; ${ }^{\Delta} P<0.01$, compared with moderate stenosis.

Table 2. There were no significant differences in TC, LDLC, apoB100, and lipoprotein(a) levels between non-CAD group and CAD group. Compared with non-CAD group, the incidences of related diseases (diabetes and hypertension) and triglycerides level were significantly higher $(P<0.01)$, whereas levels of HDL-C and apoAI, HDL-C/LDL-C ratio, and apoAI/apoB100 ratio were significantly lower $(P<$ 0.01 ) in the CAD group. When the severity of CAD was evaluated by angiographic GSS, no statistically significant differences in classic risk factors, except for apoAI level, were observed between the groups with different extent of CAD. Meanwhile, we examined plasma hs-CRP as an inflammatory biomarker in all subjects, and our result showed that hs-CRP level in CAD patients was $2.40(0.99 \sim 6.17) \mathrm{mg} / \mathrm{L}$ which was significantly higher than that of non-CAD group $(P<0.01)$. Highest hs-CRP level appeared in severe CAD group, but there was no significant difference in hs-CRP level between mild, moderate CAD, and non-CAD groups.

Interestingly, PON1 activity in different CAD groups was significantly lower than that of non-CAD patient group $(P<$ 0.01 ), exhibiting a close relationship with the risk of CAD. The decreases of PON1 activity in severe CAD group were especially evident compared with those in mild or moderate CAD group $(P<0.01)$, while subjects with mild and moderate CAD had comparable PON1 activities. The linear regression analysis revealed a significant correlation between PON1 activity and the severity of CAD as assessed by GSS $(r$ $=-0.393, P<0.001)$ (Figure 2).

The relationship between PON1 activity and clinical or biochemical parameters was also examined. The analysis by Pearson or Spearman correlation showed that PON1 activities in all subjects were positively associated with HDL-C $(r=$ 


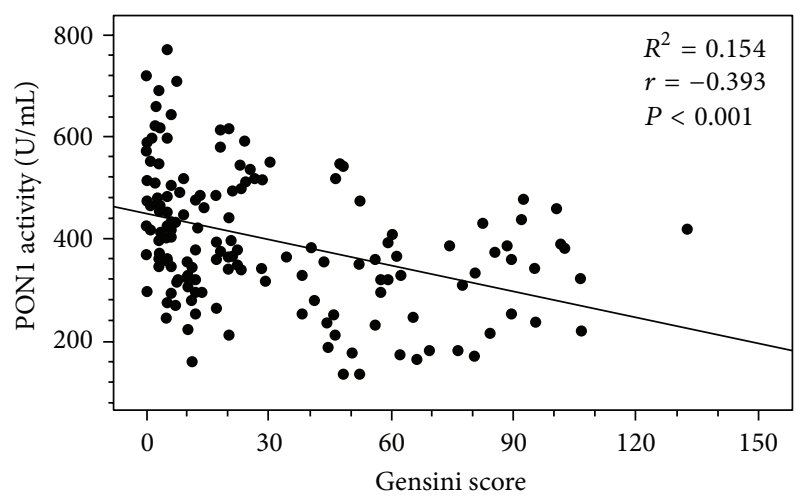

Figure 2: Correlation between PON1 activity and the severity of atherosclerosis in patients. The linear regression analysis revealed a significant correlation between PON1 activity and the severity of atherosclerosis as assessed by the angiographic Gensini score (GSS) (Pearson correlation coefficient $r=-0.393$, associated $P<0.001$ ).

$0.242, P<0.01)$ and apoAI $(r=0.220, P<0.01)$, while negatively associated with age $(r=-0.218, P<0.01)$, sex $(r=-0.186, P<0.05)$, CAD $(r=-0.409, P<0.001)$, diabetes $(r=-0.267, P=0.001)$, and hs-CRP level $(r=$ $-0.216, P<0.01)$. The difference in PON1 activity was found to be independent of smoking, hypertension, TC, LDL-C, apoB100, and lipoprotein(a) concentrations in the studied subjects (Table 3 ).

\section{Discussion}

More than 95\% of PON1 is associated with HDL particles in the circulation. PON1 may contribute substantially to the antiatherogenic properties of HDL through its well-established anti-oxidant and anti-inflammatory role by hydrolyzing oxidized lipids $[4,18]$. In addition, PON1, as a lactonase, has the ability to detoxify homocysteine(hcy-) thiolactone and to minimize protein damage by $\mathrm{N}$ homocysteinylation, which is an independent risk factor of atherosclerosis [19]. There is increasing evidence linking lower PON1 activity in serum or plasma to an increased likelihood of CAD $[10,11]$. The main finding of our study was that PON1 activity is reversely correlated with the severity of CAD.

$\mathrm{ApoE}^{-/-}$mice develop severe hypercholesterolemia and spontaneous atherosclerosis with age. The mice fed a chow diet exhibited increased plasma TC (5-8-fold) and triglycerides (1.7-fold) compared to C57BL6 mice, the complexity of the progressive lesions and is similar to that described in humans, therefore representing an important model to study the influence of genetic and environmental factors on the atherogenic process $[20,21]$. In present study, using female $\mathrm{apoE}^{-1-}$ mice of different ages fed a regular chow diet, we found that hypercholesterolemia was significantly aggravated in elder mice, and in these mice progressive atherosclerotic lesions were obviously observed in the aortic root and throughout the arterial tree compared to those of 3-month-old mice. It is striking that serum PON1 activity in
TABLE 3: Correlation between paraoxonase-1 activity and other factors.

\begin{tabular}{lcc}
\hline Variables & $r$ & PON1 activity \\
\hline Age & -0.218 & $0.008^{* *}$ \\
Sex & -0.186 & $0.023^{*}$ \\
Smoking & 0.066 & 0.422 \\
CAD & -0.409 & $<0.000^{* *}$ \\
Diabetes & -0.267 & $0.001^{* *}$ \\
Hypertension & -0.088 & 0.285 \\
Triglycerides & 0.006 & 0.944 \\
TC & 0.057 & 0.492 \\
HDL-C & 0.242 & $0.003^{* *}$ \\
LDL-C & 0.042 & 0.610 \\
ApoAI & 0.220 & $0.007^{* *}$ \\
ApoB100 & 0.082 & 0.317 \\
Lipoprotein(a) & -0.027 & 0.744 \\
hs-CRP & -0.216 & $0.008^{* *}$ \\
\hline${ }^{*} P<0.05 ;{ }^{* *} P<0.01$. & &
\end{tabular}

${ }^{*} P<0.05 ;{ }^{* *} P<0.01$.

apoE $\mathrm{E}^{-/-}$mice gradually decreased with age and was reversely correlated with the severity and extent of atherosclerotic lesions. We also found that lower PON1 activity was accompanied with elevated levels of inflammatory cytokines, such as TNF- $\alpha$ and IL6, suggesting that the decrease in PON1 activity may occur as integral part of an inflammatory response. It has been reported that inflammatory conditions result in PON1 enzymatic activity exhaustion and downregulation of hepatic PON1 mRNA levels [22, 23].

We further explored the relationship between PON1 activity and the extent of atherosclerosis in angiographically proven CAD patients. In clinic, invasive coronary angiography is the gold standard for diagnosis of obstructive CAD and also useful for evaluation of the severity of CAD according to the degree of luminal stenosis using the Gensini score (GSS) [15]. Similar to the previous findings [10,11], our data showed that PON1 activity was significantly lower in CAD patients than non-CAD patients; furthermore, the regression analysis revealed a strong reverse correlation between PON1 activity and the severity of CAD reflected by GSS in Chinese subjects.

Interestingly, the present data indicate that the etiology of atherosclerosis in the mouse model is different from the etiology of CAD in the human study. Elevated TC level is strongly correlated with the severity of atherosclerosis in apoE ${ }^{-/-}$mice. In human study, we did not find significant difference in TC and LDL-C levels between non-CAD group and CAD group; however, HDL-C level and HDL-C/LDL-C ratio were significantly lower in the $\mathrm{CAD}$ group. In addition, plasma triglycerides level was significantly higher in the CAD group. According to the recent AHA scientific statement, increase in triglycerides level is a critical risk factor for development of cardiovascular disease [24], so it is likely that multiple risk factors are responsible for the development of CAD in this population, such as decrease in plasma HDL$\mathrm{C}$ level and increase in triglycerides level and inflammatory status. 
Although the mechanism of PON1 regulation is currently not well understood, there is considerable evidence that PON1 activity is influenced by genetic and environmental factors, such as age, gender, smoking, alcohol consumption, fatrich diet, and drugs [13, 25]. For example, the PON1-R192Q polymorphism is found to correlate with PON1 activity, in which the R192 allele gives rise to a higher PON1 activity $[13,25]$. We have tested the PON1-R192Q polymorphism in a small number of subjects to whom DNA samples are available for genotyping in both control and CAD groups. There is no significant difference in the $\mathrm{R}$ allele frequency between the two groups. In the present study, while the subjects had no difference in drug use, we observed that those well-known traditional risk factors of atherosclerosis, such as age, sex, diabetes, and plasma HDL-C and apoAI levels, were significantly correlated with PON1 activity (Table 3 ). These were in agreement with the previous findings [26]. However, there are also some reports showing that changes in PON1 activity might occur independently of changes in levels of HDL-C and apoAI $[27,28]$. We suggest that PON1 and HDL$\mathrm{C}$ or apoAI as a whole HDL particle may be influenced by the same determinants, whereas it is possible that PON1 activity is affected by the other covariates or more sensitive to certain conditions.

Our efforts were focused on how PON1 activity is modulated by inflammation, since inflammation plays a crucial role in the pathogenesis of atherosclerotic CAD. HDL may be converted from an anti-inflammatory to a proinflammatory particle when PON1 level or activity associated with HDL is diminished during the acute phase response $[1,29,30]$. It has been documented that inflammatory markers such as CRP are directly involved in the atherosclerotic process and may serve as predictor for the risk of CAD [31,32]. Moreover, hsCRP has a generally reverse correlation with PON1 activity in diabetes patients with coronary heart disease [33]. Our result also demonstrated that patients with CAD exhibited increased hs-CRP levels in circulation. In addition, we found that PON1 activity was significantly associated with the level of hs-CRP, providing a mechanistic link between inflammation and decreased PON1 activity in the development of atherosclerosis. Interestingly, when a subgroup analysis among CAD patients was performed, we found that PON1 activity was closely correlated with the severity of coronary stenosis, but no statistically significant differences of hs-CRP levels were observed between mild CAD, moderate CAD, and non-CAD groups.

Our data are in agreement with the concept that atherosclerosis is a multifactorial, complex, chronic developing metabolic disease. Dyslipidemia in combination with inflammation initiates and aggravates the development of atherosclerosis and CAD [1]. Therefore, to explore the sensitive markers associated with HDL such as PON1 activity is important for the evaluation of antioxidative or antiinflammatory properties of HDL and of its role in the progression of cardiovascular disease [34]. Previous work using a PON1 gene knockout mouse model showed that these mice had a significant vascular inflammatory tendency and increasing lipoprotein oxidation and developed atherosclerosis faster but had no significant alteration in plasma lipid profile, when compared with control mice $[6,7]$. Recently, Fuhrman et al. reported that PON1 deficiency in mice resulted in reduced macrophage SR-BI expression, decreased cellular HDL binding, and loss of HDL-mediated cytoprotection against apoptosis, providing new insights suggesting that reduced PON1 activity could promote HDL dysfunction and diminish cytoprotective capacity of HDL [35]. Besler et al. also showed that reduced PON1 activity in CAD patients may be responsible for loss of the anti-inflammatory and repair-stimulating effects of HDL on endothelium [36]. It is convincible that $\mathrm{PON} 1$ not only has a main role in inactivation of LDL oxidation during the initiation of atherosclerosis, but also promotes HDL-mediated cytoprotection during the development of atherosclerosis. Our study supports that PON1 activity is affected by a variety of factors, for example, inflammatory status, and that decrease of PON1 activity further aggravates HDL dysfunction during the progressive atherosclerosis, suggesting that PON1 activity may serve as a potential biomarker for the severity of atherosclerotic CAD.

In conclusion, PON1, as an antioxidative and antiinflammatory component of $\mathrm{HDL}$, plays an important role in halting atherogenesis. Our experimental and clinical evidence demonstrates that the decrease of PON1 activity is accompanied with the increase of inflammatory status and is closely correlated with the progression of atherosclerosis. Thus, the present findings suggest that the lower PON1 activity may be an important inflammatory indicator for the severity of atherosclerosis and CAD events. Enhancing PON1 activity could be a promising strategy for reducing $\mathrm{CAD}$ risk and preventing the progression of cardiovascular atherosclerosis.

\section{Authors' Contribution}

Chiyan Zhou and Jia Cao contributed equally to this work.

\section{Acknowledgments}

This work was supported by the Grants from National Natural Science Foundation of China (nos. 30770850 and 81201335) and the Grant from the Scientific and Technological Ministry of Hubei province, China (nos. 2007ABB024 and 2009CDA079).

\section{References}

[1] G. K. Hansson, "Mechanisms of disease: inflammation, atherosclerosis, and coronary artery disease," The New England Journal of Medicine, vol. 352, no. 16, pp. 1685-1695, 2005.

[2] N. R. Madamanchi, A. Vendrov, and M. S. Runge, "Oxidative stress and vascular disease," Arteriosclerosis, Thrombosis, and Vascular Biology, vol. 25, no. 1, pp. 29-38, 2005.

[3] F. Tabet and K.-A. Rye, "High-density lipoproteins, inflammation and oxidative stress," Clinical Science, vol. 116, no. 2, pp. 8798, 2009.

[4] G. S. Getz and C. A. Reardon, "Paraoxonase, a cardioprotective enzyme: continuing issues," Current Opinion in Lipidology, vol. 15 , no. 3, pp. 261-267, 2004. 
[5] B. MacKness and M. MacKness, "Anti-inflammatory properties of paraoxonase-1 in atherosclerosis," Advances in Experimental Medicine and Biology, vol. 660, pp. 143-151, 2010.

[6] D. M. Shih, Y.-R. Xia, X.-P. Wang et al., "Combined serum paraoxonase knockout/apolipoprotein E knockout mice exhibit increased lipoprotein oxidation and atherosclerosis," Journal of Biological Chemistry, vol. 275, no. 23, pp. 17527-17535, 2000.

[7] D. S. Ng, T. Chu, B. Esposito, P. Hui, P. W. Connelly, and P. L. Gross, "Paraoxonase-1 deficiency in mice predisposes to vascular inflammation, oxidative stress, and thrombogenicity in the absence of hyperlipidemia," Cardiovascular Pathology, vol. 17, no. 4, pp. 226-232, 2008.

[8] A. Tward, Y.-R. Xia, X.-P. Wang et al., "Decreased atherosclerotic lesion formation in human serum paraoxonase transgenic mice," Circulation, vol. 106, no. 4, pp. 484-490, 2002.

[9] B. Mackness, R. Quarck, W. Verreth, M. Mackness, and P. Holvoet, "Human paraoxonase-1 overexpression inhibits atherosclerosis in a mouse model of metabolic syndrome," Arteriosclerosis, Thrombosis, and Vascular Biology, vol. 26, no. 7, pp. 1545-1550, 2006.

[10] M. Wang, X. Lang, S. Cui et al., "Quantitative assessment of the influence of paraoxonase 1 activity and coronary heart disease risk," DNA and Cell Biology, vol. 31, pp. 975-982, 2012.

[11] Y. Zhao, Y. Ma, Y. Fang et al., "Association between PON1 activity and coronary heart disease risk: a meta-analysis based on 43 studies," Molecular Genetics and Metabolism, vol. 105, no. 1, pp. 141-148, 2012.

[12] Z. Tartan, G. Orhan, H. Kasikçioglu et al., "The role of paraoxonase (PON) enzyme in the extent and severity of the coronary artery disease in type- 2 diabetic patients," Heart and Vessels, vol. 22, no. 3, pp. 158-164, 2007.

[13] A. Balcerzyk, I. Zak, and J. Krauze, "Protective effect of R allele of PON1 gene on the coronary artery disease in the presence of specific genetic background," Disease Markers, vol. 24, no. 2, pp. 81-88, 2008.

[14] B. Thyagarajan, D. R. Jacobs Jr., J. J. Carr et al., "Factors associated with paraoxonase genotypes and activity in a diverse, young, healthy population: the Coronary Artery Risk Development in Young Adults (CARDIA) study," Clinical Chemistry, vol. 54, no. 4, pp. 738-746, 2008.

[15] G. G. Gensini, "A more meaningful scoring system for determining the severity of coronary heart disease," American Journal of Cardiology, vol. 51, no. 3, p. 606, 1983.

[16] K. Handa, S. Kono, K. Saku et al., "Plasma fibrinogen levels as an independent indicator of severity of coronary atherosclerosis," Atherosclerosis, vol. 77, no. 2-3, pp. 209-213, 1989.

[17] H. Yu, W. Zhang, P. G. Yancey et al., "Macrophage apolipoprotein $\mathrm{E}$ reduces atherosclerosis and prevents premature death in apolipoprotein $\mathrm{E}$ and scavenger receptor-class BI doubleknockout mice," Arteriosclerosis, Thrombosis, and Vascular Biology, vol. 26, no. 1, pp. 150-156, 2006.

[18] V. G. Cabana, C. A. Reardon, N. Feng, S. Neath, J. Lukens, and G. S. Getz, "Serum paraoxonase: effect of the apolipoprotein composition of HDL and the acute phase response," Journal of Lipid Research, vol. 44, no. 4, pp. 780-792, 2003.

[19] J. Perla-Kajan and H. Jakubowski, "Paraoxonase 1 and homocysteine metabolism," Amino Acids, vol. 43, no. 4, pp. 1405-1417, 2012.

[20] A. S. Plump, J. D. Smith, T. Hayek et al., "Severe hypercholesterolemia and atherosclerosis in apolipoprotein E-deficient mice created by homologous recombination in ES cells," Cell, vol. 71, no. 2, pp. 343-353, 1992.
[21] S. H. Zhang, R. L. Reddick, J. A. Piedrahita, and N. Maeda, "Spontaneous hypercholesterolemia and arterial lesions in mice lacking apolipoprotein E," Science, vol. 258, no. 5081, pp. 468471, 1992.

[22] K. R. Feingold, R. A. Memon, A. H. Moser, and C. Grunfeld, "Paraoxonase activity in the serum and hepatic mRNA levels decrease during the acute phase response," Atherosclerosis, vol. 139, no. 2, pp. 307-315, 1998.

[23] J. Kotur-Stevuljevic, S. Spasic, Z. Jelic-Ivanovic et al., "PON1 status is influenced by oxidative stress and inflammation in coronary heart disease patients," Clinical Biochemistry, vol. 41, no. 13, pp. 1067-1073, 2008.

[24] M. Miller, N. J. Stone, C. Ballantyne et al., “Triglycerides and cardiovascular disease: a scientific statement from the American Heart Association," Circulation, vol. 123, no. 20, pp. 2292-2333, 2011.

[25] D. L. Rainwater, S. Rutherford, T. D. Dyer et al., "Determinants of variation in human serum paraoxonase activity," Heredity, vol. 102, no. 2, pp. 147-154, 2009.

[26] M.-C. Blatter Garin, X. Moren, and R. W. James, "Paraoxonase1 and serum concentrations of HDL-cholesterol and apoA-I," Journal of Lipid Research, vol. 47, no. 3, pp. 515-520, 2006.

[27] L. S. Rozek, T. S. Hatsukami, R. J. Richter et al., "The correlation of paraoxonase (PON1) activity with lipid and lipoprotein levels differs with vascular disease status," Journal of Lipid Research, vol. 46, no. 9, pp. 1888-1895, 2005.

[28] P. D. Sarkar, T. M. Shivaprakash, and B. Madhusudhan, "Association between paraoxonase activity and lipid levels in patients with premature coronary artery disease," Clinica Chimica Acta, vol. 373, no. 1-2, pp. 77-81, 2006.

[29] A. Kontush and M. J. Chapman, "Functionally defective highdensity lipoprotein: a new therapeutic target at the crossroads of dyslipidemia, inflammation, and atherosclerosis," Pharmacological Reviews, vol. 58, no. 3, pp. 342-374, 2006.

[30] M. Navab, S. T. Reddy, B. J. van Lenten, and A. M. Fogelman, "HDL and cardiovascular disease: atherogenic and atheroprotective mechanisms," Nature Reviews Cardiology, vol. 8, no. 4, pp. 222-232, 2011.

[31] Y. Zhao, R. Wang, X. Ma et al., "Distribution of C-reactive protein and its association with cardiovascular risk factors in a population-based sample of Chinese," Disease Markers, vol. 28, no. 6, pp. 333-342, 2010.

[32] N. Sarwar, A. J. Thompson, and E. Di Angelantonio, "Markers of inflammation and risk of coronary heart disease," Disease Markers, vol. 26, no. 5-6, pp. 217-225, 2009.

[33] B. Mackness, D. Hine, P. McElduff, and M. Mackness, "High Creactive protein and low paraoxonasel in diabetes as risk factors for coronary heart disease," Atherosclerosis, vol. 186, no. 2, pp. 396-401, 2006.

[34] C. Mineo and P. W. Shaul, "PON-dering differences in HDL function in coronary artery disease," Journal of Clinical Investigation, vol. 121, no. 7, pp. 2545-2548, 2011.

[35] B. Fuhrman, A. Gantman, and M. Aviram, "Paraoxonase 1 (PON1) deficiency in mice is associated with reduced expression of macrophage SR-BI and consequently the loss of HDL cytoprotection against apoptosis," Atherosclerosis, vol. 211, no. 1, pp. 61-68, 2010.

[36] C. Besler, K. Heinrich, L. Rohrer et al., "Mechanisms underlying adverse effects of HDL on eNOS-activating pathways in patients with coronary artery disease," Journal of Clinical Investigation, vol. 121, no. 7, pp. 2693-2708, 2011. 


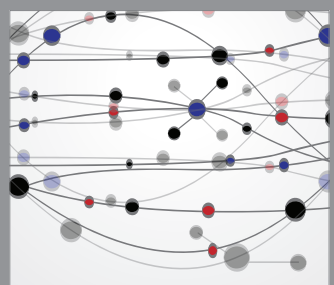

The Scientific World Journal
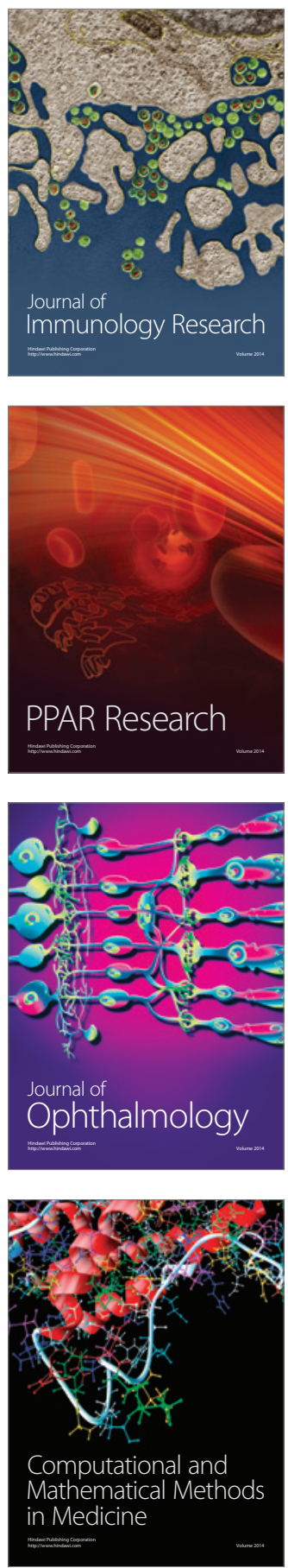

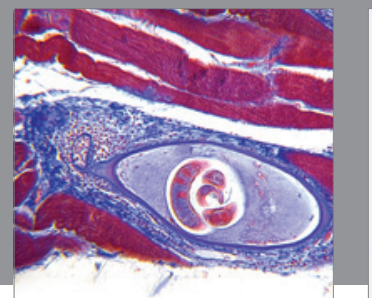

Gastroenterology

Research and Practice
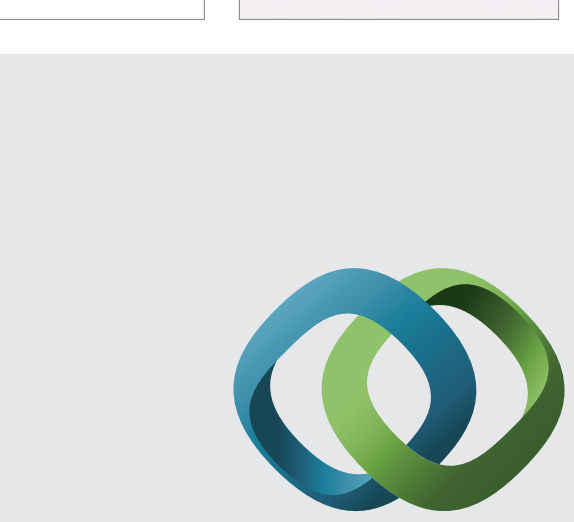

\section{Hindawi}

Submit your manuscripts at

http://www.hindawi.com
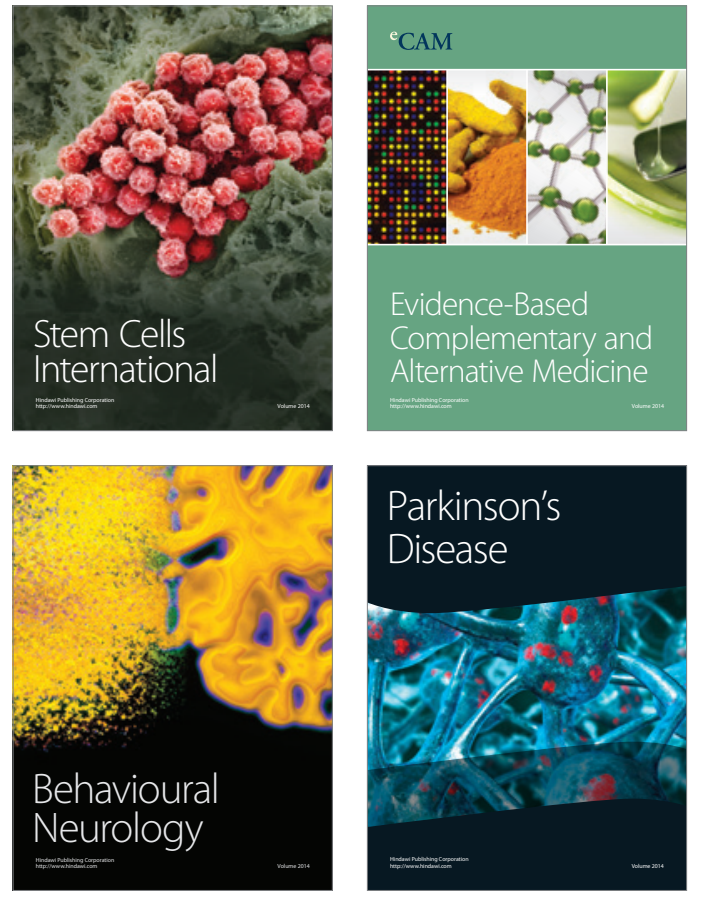
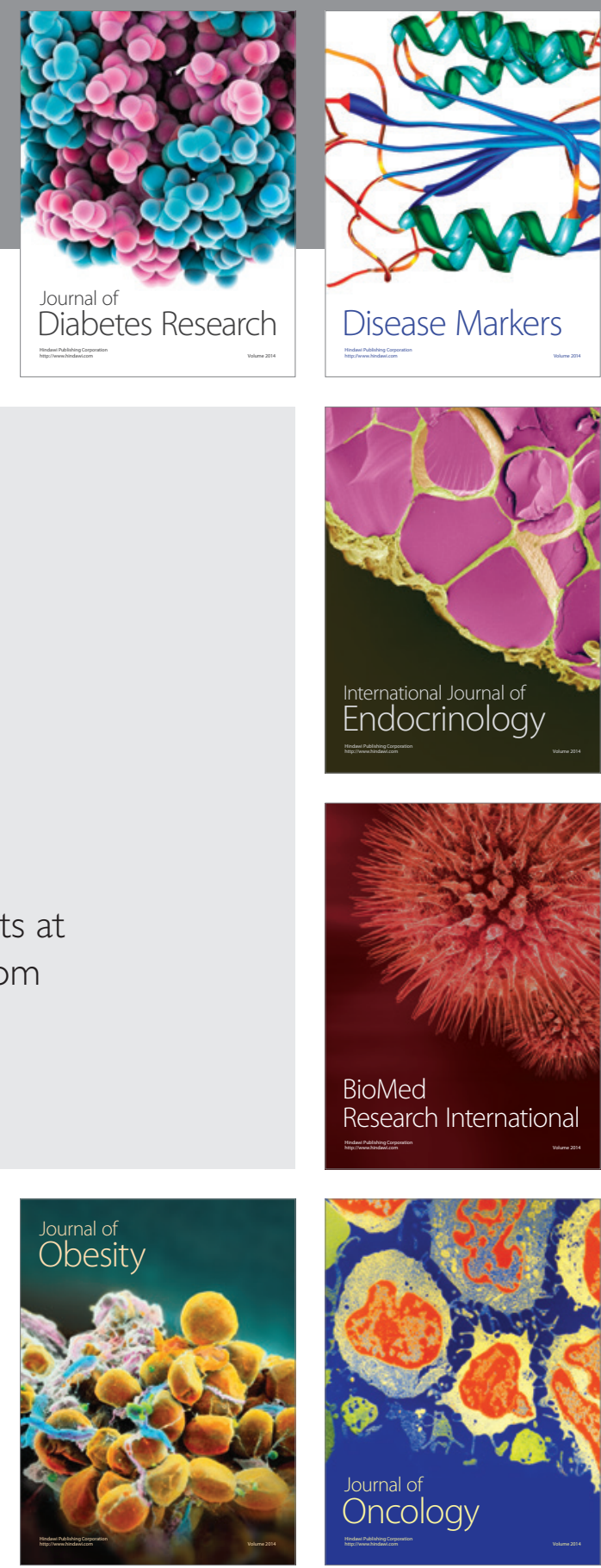

Disease Markers
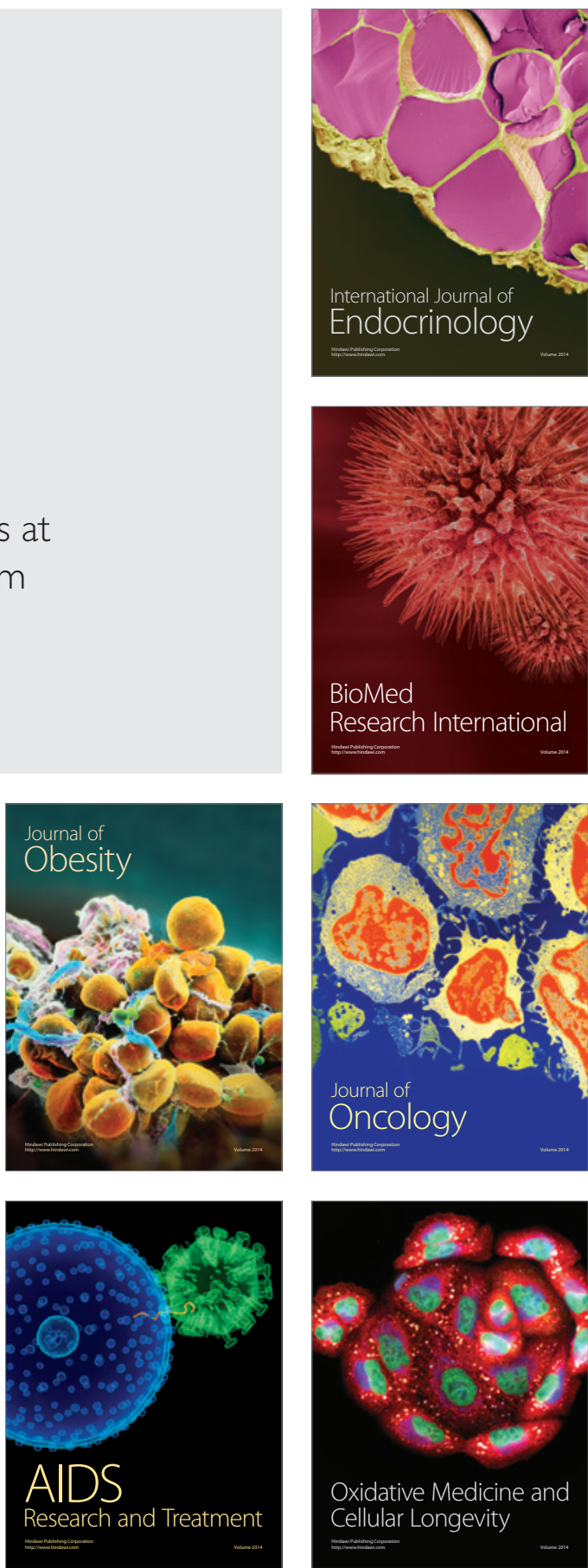